\title{
Operation of X-ray gas monitors at the European XFEL
}

\author{
Theophilos Maltezopoulos, Florian Dietrich, Wolfgang Freund, Ulf Fini \\ Jastrow, Andreas Koch, Joakim Laksman, Jia Liu, Marc Planas, Andrey A. \\ Sorokin, Kai Tiedtke and Jan Grünert
}

J. Synchrotron Rad. (2019). 26, 1045-1051

\section{IUCr Journals CRYSTALLOGRAPHY JOURNALS ONLINE}

Copyright (C) International Union of Crystallography

Author(s) of this article may load this reprint on their own web site or institutional repository provided that this cover page is retained. Republication of this article or its storage in electronic databases other than as specified above is not permitted without prior permission in writing from the IUCr.

For further information see https://journals.iucr.org/services/authorrights.html 
JOURNAL OF SYNCHROTRON RADIATION
Received 4 January 2019

Accepted 19 March 2019

Edited by M. Zangrando, IOM-CNR and Elettra-Sincrotrone, Italy

${ }^{1}$ This article will form part of a virtual special issue containing papers presented at the PhotonDiag2018 workshop.

Keywords: free-electron lasers; photon diagnostics; hard X-rays.

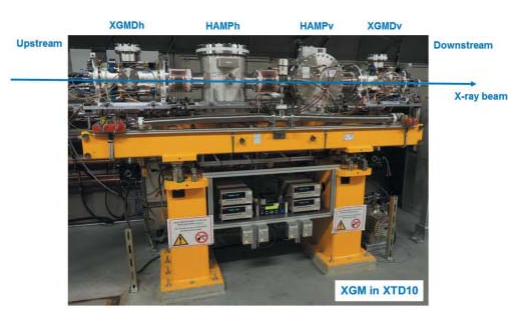

(C) 2019 International Union of Crystallography

\section{Operation of X-ray gas monitors at the European XFEL $^{1}$}

\author{
Theophilos Maltezopoulos, ${ }^{\text {a* }}$ Florian Dietrich, ${ }^{\mathrm{a}}$ Wolfgang Freund, ${ }^{\mathrm{a}}$

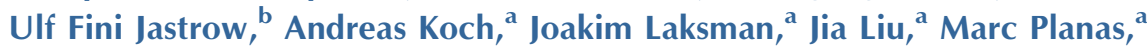 \\ Andrey A. Sorokin,, ${ }^{b, c}$ Kai Tiedtke ${ }^{b}$ and Jan Grünert ${ }^{a}$ \\ ${ }^{a}$ X-ray Photon Diagnostics Group, European XFEL, Holzkoppel 4, 22869 Schenefeld, Germany,

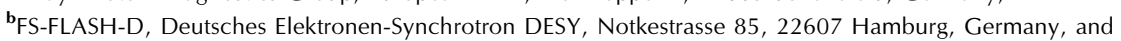 \\ ' Ioffe Physico-Technical Institute, Polytekhnicheskaya 26, 194021 St Petersburg, Russian Federation. \\ *Correspondence e-mail: theophilos.maltezopoulos@xfel.eu
}

X-ray gas monitors (XGMs) are operated at the European XFEL for noninvasive single-shot pulse energy measurements and average beam position monitoring. They are used for tuning and maintaining the self-amplified spontaneous emission (SASE) operation and for sorting single-shot experimental data according to the pulse-resolved energy monitor data. The XGMs were developed at DESY based on the specific requirements for the European XFEL. In total, six XGM units are continuously in operation. Here, the main principle and experimental setup of an XGM are summarized, and the locations of the six XGMs at the facility are shown. Pulse energy measurements at $0.134 \mathrm{~nm}$ wavelength are presented, exceeding $1 \mathrm{~mJ}$ obtained with an absolute measurement uncertainty of 7-10\%; correlations between different XGMs are shown, from which a SASE1 beamline transmission of $97 \%$ is deduced. Additionally, simultaneous position measurements close to the undulator and at the end of the tunnel are shown, along with the correlation of beam position data simultaneously acquired by an XGM and an imager.

\section{Introduction}

Free-electron lasers (FELs) which are operated in the selfamplified spontaneous emission mode (SASE) are currently the most intense femtosecond (fs) light sources from the extreme ultraviolet down to the hard X-rays wavelength regime. However, the statistical nature of the SASE process leads to intensity and spectral fluctuations in the photon pulses (Saldin et al., 2000). Moreover, the electron acceleration process introduces arrival-time fluctuations to the electron bunches, which leads in pump-probe experiments to a temporal jitter of the photon pulses at the scientific endstations with respect to a synchronized external optical laser. Therefore, several diagnostic tools have been developed in order to measure pulse by pulse the photon energy (Richter $e t$ al., 2003; Sorokin, 2004), temporal profile (Frühling et al., 2009) and arrival time (Maltezopoulos et al., 2008) of the SASE pulses. In addition, for measuring the spectrum and polarization of the photon pulses the photoelectron spectrometer (PES) (Buck, 2012; Lutman et al., 2016) can be used. This gas-based online method measures angular resolved the time-of-flight of photoelectrons [for further details see Laksman et al. (2019)]. With these pulse tagging approaches, experimental data can be sorted and categorized.

Gas monitor detectors (GMDs) were developed at the freeelectron laser in Hamburg (FLASH) (Richter et al., 2003; Sorokin, 2004; Tiedtke et al., 2008; Saito et al., 2010; Kato et al., 
2010) to measure non-invasively the single-shot photon pulse energy and to monitor the average beam position in the extreme ultraviolet wavelength (XUV) range. Meanwhile, many facilities worldwide have used different attempts for gasbased online intensity diagnostics (Moeller et al., 2011; Kato et al., 2012; Tiedtke et al., 2014; Song et al., 2019). With the development of X-ray gas monitors (XGMs), the GMD technique was extended to X-ray photon energies (Sorokin et al., 2011, 2019; Kato et al., 2012; Tiedtke et al., 2014).

The measurement principle is based on photo-ionization of rare gas atoms; therefore, it is not degrading during operation and, thanks to the replenishing interaction sample, is almost transparent. The photo-ion current is absolutely calibrated to the photon flux at the Physikalisch-Technische Bundesanstalt (PTB). XGMs can measure individual femtosecond X-ray pulses containing $10^{7}$ up to $10^{15}$ photons with an absolute measurement accuracy of $7-10 \%$. The average beam position measurement accuracy is of the order of $\pm 10 \mu \mathrm{m}$ within a range of $\pm 1 \mathrm{~mm}$. The average beam position measurement linearity was verified for a range of $\pm 6 \mathrm{~mm}$.

In this paper we will present the operation of XGMs at the European XFEL. This facility operates in a burst mode, where the burst train repetition rate is $10 \mathrm{~Hz}$. One pulse train can contain from 1 up to 2700 pulses within a time window of $600 \mu \mathrm{s}$. The highest intra pulse train repetition rate is $4.5 \mathrm{MHz}$ and the lowest is $1.7 \mathrm{kHz}$ (here only two pulses per train are possible). After an introduction of the operation principle and experimental setup, we will show the locations of our six XGMs in the facility, and present experimental data from commissioning and the first year of user operation.

\section{Instrumentation}

A photograph of an XGM installed in the XTD10 tunnel is shown in Fig. 1. The XGM consists of four chambers, named XGMDh, HAMPh, HAMPv and XGMDv. The X-ray gas monitor detector (XGMD) is a development performed by Deutsches ElektronenSynchrotron (DESY) towards hard X-rays based on the XUV version of the GMD. The Huge Aperture Open Multiplier (HAMP) is the most recent development to enable single-shot resolution even using very hard X-rays. Since we are also interested in beam position monitoring, each XGM contains both types of chambers twice, rotated around the beam propagation by $90^{\circ}$ with respect to each other: chambers ending in $\mathrm{h}$ or $\mathrm{v}$ stand for horizontal or vertical beam position monitoring, respectively.

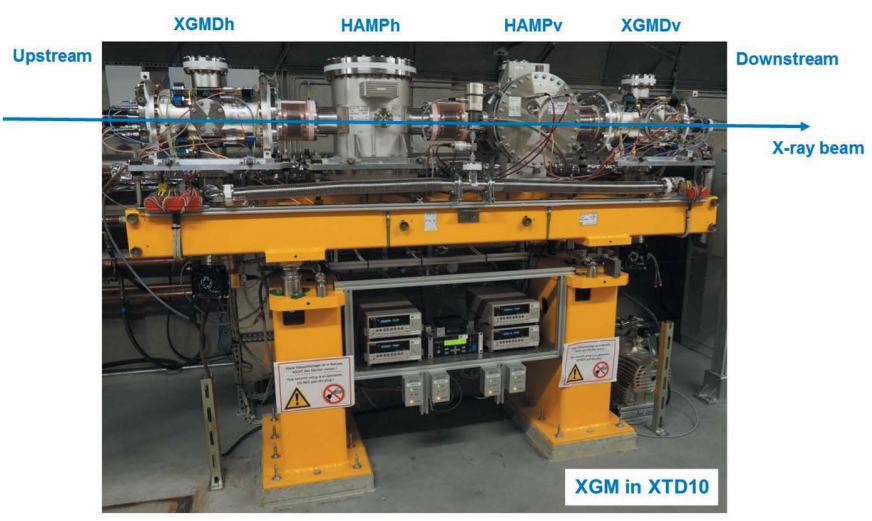

Figure 1

Photograph of the XGM in the XTD10 tunnel shown as an example. The XGM consists of four chambers: XGMDh, HAMPh, HAMPv and XGMDv. Chamber names ending with the letters $\mathrm{h}$ or $\mathrm{v}$ are dedicated for horizontal or vertical beam position monitoring, respectively.

A detailed description of the XGMD and HAMP is given by Sorokin et al. (2011) and a new contribution is to be published (Sorokin et al., 2019). In the following we will summarize the main operation principle of XGMD and HAMP.

A schematic view of the XGMD is shown in Fig. 2. Photoions and electrons are extracted in opposite directions by an electric field, which must be sufficiently high to prevent the photoelectrons from reaching the ion plates. The photo-ions
XGMD
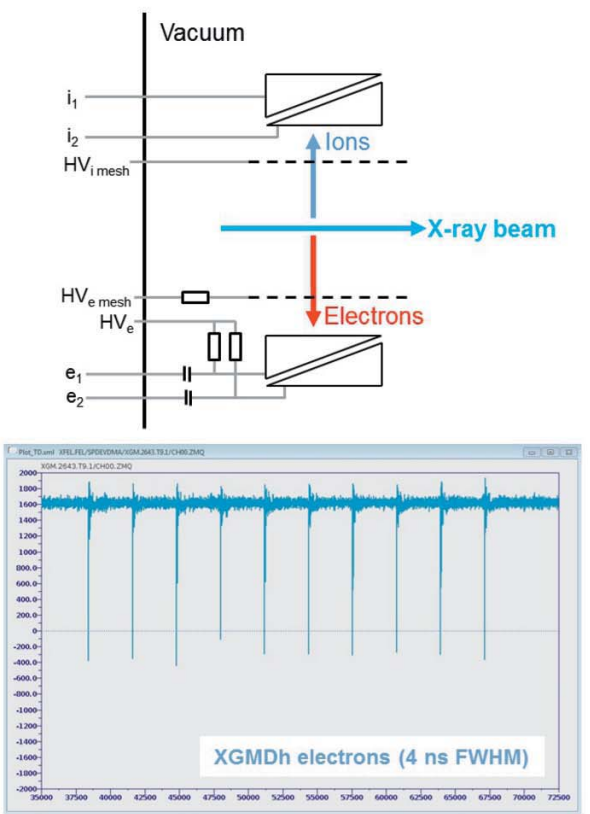

Figure 2

Sketch of the XGMD (left) and HAMP (right). The XGMD is based on photo-ionization of dilute gases. Photo-ions and electrons are extracted by an electric field $\left(\mathrm{HV}_{i \text { mesh }}, \mathrm{HV}_{\mathrm{e} \text { mesh }}, \mathrm{HV}_{\mathrm{e}}\right)$. The photoelectron signals $\left(e_{1}\right.$ and $\left.e_{2}\right)$ are coupled out over capacitors. The photo-ion currents $\left(i_{1}\right.$ and $\left.i_{2}\right)$ are coupled out directly. The plates for position monitoring are depicted schematically. HAMP is also based on the detection of photo-ions. A repeller $\left(\mathrm{HV}_{\text {rep }}\right)$ accelerates the photo-ions towards a multiplier (MP stage). This multiplier $\left(\mathrm{HV}_{\mathrm{HAMP}}\right)$ amplifies the ionic signal to an electron signal, which is coupled out of the vacuum chamber $\left(h_{1}\right.$ and $\left.h_{2}\right)$. Raw ADC traces for XGMD and HAMP signals are shown below (ten pulses per train; peak widths are indicated). 
are detected with Faraday cups and measured as low pass filtered average ion-currents $\left(i_{1}\right.$ and $i_{2}$ ) with a bandwidth of about $15 \mathrm{mHz}$ which corresponds to a time constant of tens of seconds. With this, the average photon flux can be determined in absolute terms, but not for single shots. In addition, the photoelectron signal $\left(e_{1}\right.$ and $\left.e_{2}\right)$ is coupled out over a capacitor (around $800 \mathrm{pF}$ ), amplified with $17 \mathrm{~dB}$ or $37 \mathrm{~dB}$ with a bandwidth of $500 \mathrm{MHz}$, and digitized with an analog-to-digital converter (ADC) from Teledyne SP Devices (model ADQ412-3G-MTCA, 12 bit, $2 \mathrm{GHz}$ bandwidth). Raw ADC traces are shown in Fig. 2. For this example, ten pulses within the pulse train were lasing and clearly all of them are resolved from shot to shot with a full width at half-maximum (FWHM) of $4 \mathrm{~ns}$. This signal is pulse resolved, but not absolutely calibrated like the photo-ion signal described above. In order to calibrate the single-shot signal, we first locate the peaks. We can work with the raw data or use a fit function. We can determine the peak amplitude or the peak integral, which are both linear to the pulse energy. Finally, the single-shot photoelectron signal is averaged by software to the intrinsic time scale of the ion current measurement and thereafter cross-calibrated to the photon flux. Note that there are always two plates for ions and electrons, as depicted schematically in Fig. 2. This geometry allows for position monitoring: if the photon beam is shifted to the right, the current on the right plate is higher than on the left plate. By measuring the difference between the currents relative to their sum, one can obtain non-invasively the beam position. This needs a further calibration factor which is obtained by geometrical calculations and can be measured by scanning the entire chamber relative to a stable beam (Sorokin et al., 2019). Since we need horizontal and vertical beam position monitoring, there are two XGMD chambers, named XGMDh and XGMDv.

HAMP is also based on photo-ionization of rare gas atoms (see the sketch in Fig. 2). A repeller separates the photoelectrons from the ions and accelerates the ions towards a multiplier. This multiplier converts the photo-ion signal to an electron signal, which is coupled out of the vacuum chamber, amplified, and digitized with an ADC (same electronics as for the XGMD signal). Raw ADC traces are shown in Fig. 2. These peaks are wider (100 ns FWHM) than the XGMD electron peaks (4 ns FWHM) but still resolvable at the maximum intra-train repetition rate of the European XFEL (4.5 MHz). HAMP is another single-shot data source which can be calibrated to the photo-ion signal of the XGMD as described above. The only difference between the HAMP and XGMD signals is that the calibration of HAMP must be repeated if the multiplier voltage is changed. Note that, like for the XGMD, there are two split plates for position monitoring. Since we need again horizontal and vertical beam position

Figure 3 monitoring there are two HAMP chambers, named HAMPh and HAMPv.

HAMP was developed for very hard X-rays where even the cross section of xenon is considerably decreased. Since we want to avoid secondary effects upon photo-ionization, like charge exchange between photo-ions and gas atoms or ionization by secondary electrons, and since we use high voltages, we cannot increase the working gas pressure above $1 \times 10^{-4}$ mbar to compensate for the diminishing signals. If many pulses are used (up to 2700 per train), the averaged XGMD ion signal stays resolvable but the single-shot XGMD electron signal strength will fall below the detection limit. Here HAMP can still resolve the weak single pulses if the multiplier voltage is set accordingly. Additionally, since HAMP at corresponding high amplification can resolve very weak signals, it is used by the machine operators during SASE search.

\section{Installation of XGMs at the European XFEL}

An overview of the entire European XFEL facility with accelerator, photon system and experiments is given by Tschentscher et al. (2017). The photon diagnostics at the European XFEL are described by Grünert et al. (2019a,b).

The XGMs are essential components in the photon diagnostics system of the facility. In total, six XGMs were produced by the Photon Diagnostics and Control team (FSFLASH-D) at DESY for the European XFEL. Five of them are located in the tunnels while one is installed at the Spectroscopy and Coherent Scattering (SCS) experimental hutch. Fig. 3 shows the locations of the XGMs at the European XFEL. After each of the three SASE undulators we have one XGM. Besides many other applications, these three XGMs in the tunnels XTD1, XTD2 and XTD10 help the machine operators to tune and maintain X-ray lasing in SASE2, SASE1 and SASE3, respectively. Not all end-stations are equipped with an entire XGM. The photon beamline to the Single Particles, Clusters and Biomolecules (SPB) and High Energy

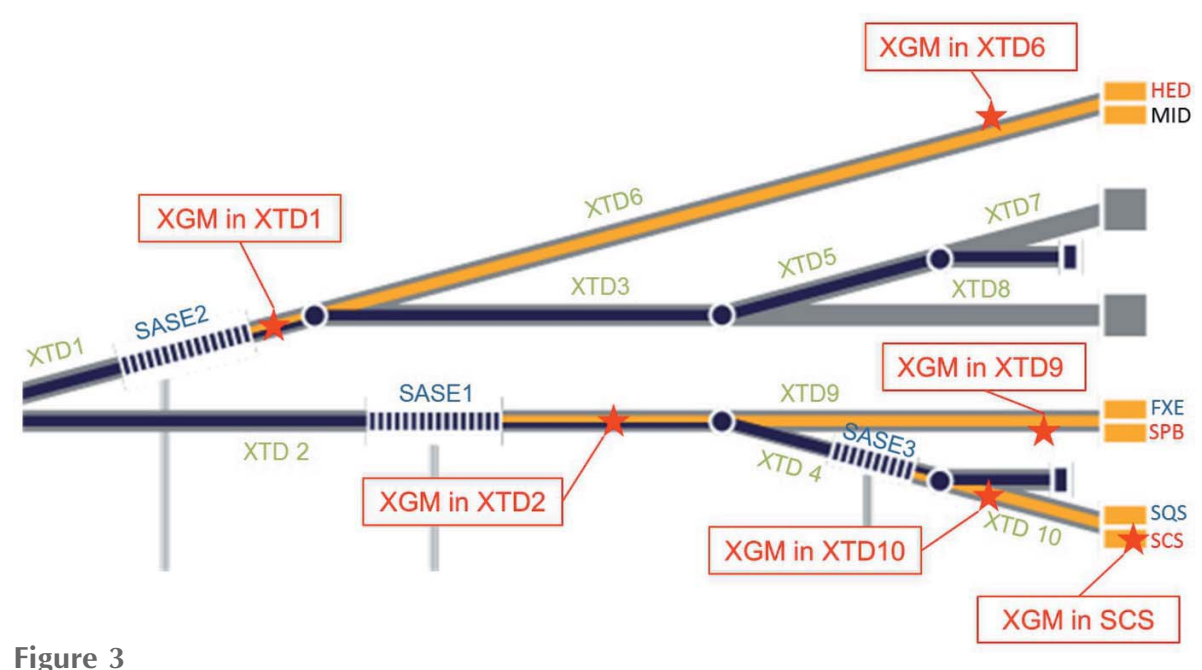

Locations of the six XGMs at the European XFEL. Five XGMs are located in the tunnels and one in the SCS experimental hutch. 
Density Matter (HED) experimental hutch in XTD9 and in XTD6, respectively, have dedicated XGMs, which measure the pulse energy and beam position at the end of the tunnels after transport through the beam optics.

Xenon, krypton, neon and nitrogen can be selected as a target gas at the XGMs in the tunnels. All gases have a purity of $5.0(99.999 \%)$. The gas cabinets are located outside the tunnels, thus bottle exchange can be carried out even during $\mathrm{X}$-ray operation independent from tunnel access times. Since process gas is injected at the XGMs into the ultra-highvacuum system, differential pumping systems (Villanueva, $2014,2016)$ are needed upstream and downstream of the instrument to maintain the otherwise lower beamline vacuum to below $10^{-8}$ mbar.

The XGM measurement controls, data acquisition, analysis and storage into the DAQ are installed in the DESY accelerator control system DOOCS. Vacuum and gas controls are operated under the European XFEL photon control system Karabo (Hauf et al., 2019). An additional DOOCS-to-Karabo bridge enables the live plot of the DOOCS data also in Karabo. Moreover, XGM data can be included in Karabo DAQ runs of users.

One big challenge is the reliable continuous operation of these instruments. Many machine parameters can vary within one shift and from one shift to another, for example the number of pulses per train, the repetition rate within the train, the photon energy and the pulse energy. The requirement for the XGMs is that they are always online, calibrated, and the evaluated energy values must be reliable. This sets high requirements to the lifetime and reliability of the subcomponents and the operation and analysis software. The XGM servers constantly read machine parameters and use them to evaluate the raw data. For example, if the photon energy changes, a new cross section and ion mean charge have to be used. If the number of pulses per train changes, the ion current has to be divided by the new value. If the intra train repetition rate changes, the fast ADC signal has to be evaluated accordingly.

\section{Results}

SASE1 is the first undulator source of the European XFEL which started operation in Spring 2017. Thereafter, SASE3 and SASE2 followed successively in the first half of 2018. Meanwhile, simultaneous lasing operation of all three SASE light sources was demonstrated.

The HAMP multiplier voltage (see Fig. 2) can be set to a corresponding high value which resolves even the weakest photon pulses. Therefore, the HAMP signal (see Fig. 2, right lower part) was used for finding the lasing signature at the SASE threshold and for optimization.

An example for stable SASE1 operation over $2 \mathrm{~h}$ is shown in Fig. 4. The left vertical axis shows the beam position (grey dots), while the right vertical axis displays the pulse energy (orange dots). Beam position and pulse energy data are deduced from the average ion signal of XGMDh and XGMDv. The horizontal axis counts the pulse train numbers with each

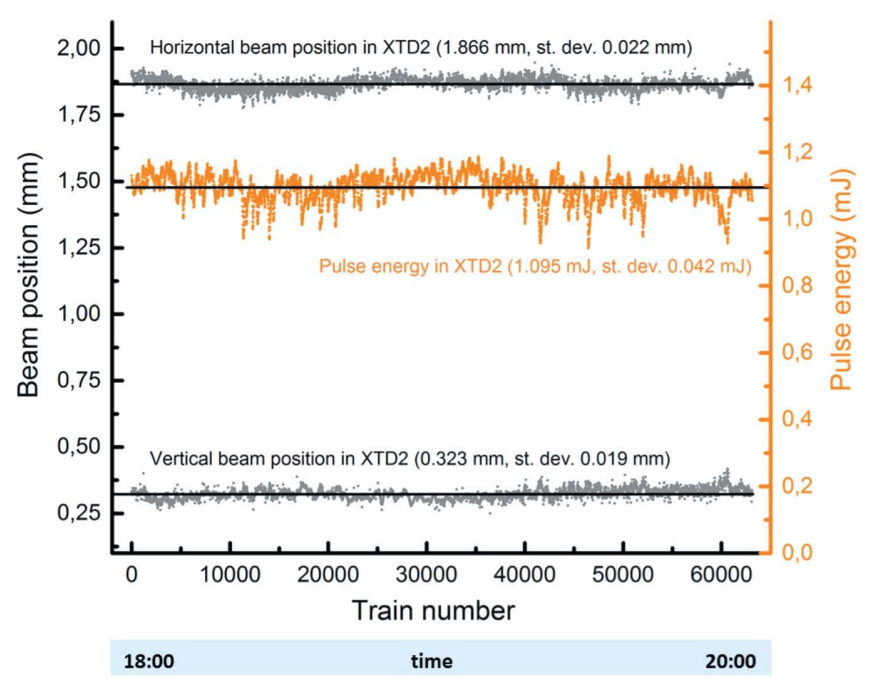

Figure 4

Pulse energy and beam position measurements over $2 \mathrm{~h}$ after SASE1 tuning and optimization. The left vertical axis is the beam position (grey dots); the right vertical axis is the pulse energy (orange dots). The data source is XTD2 XGM (SASE1), averaged ion signal of XGMDh and XGMDv, date 4 December 2017, wavelength $0.133 \mathrm{~nm}, 30$ pulses per train, with a Xe gas target. The absolute measurement uncertainty $\sigma$ of the energy was $7-10 \%(77-110 \mu \mathrm{J})$.

train consisting of 30 single shots. The conventions for the XGM beam positions are: positive values in the horizontal direction correspond to a beam position in the beam direction to the right, and positive values in the vertical direction correspond to a beam position in the beam direction upwards. Thus, in this example the beam position relative to the XGM centre is almost at the centre in the vertical direction, but horizontally it is $1.9 \mathrm{~mm}$ in the beam direction to the right. According to beam position calibration experiments, a deviation of up to $\pm 6 \mathrm{~mm}$ in the horizontal or vertical direction can be reliably measured (Sorokin et al., 2019). For larger beam offsets relative to the XGM chamber centre, the linear calibration value which is used for position deduction is no longer sufficient for precise position monitoring.

The XGMs in XTD2 and XTD9, see Fig. 3, can measure simultaneously the SASE1 beam, if the SPB branch is selected for operation. A linear correlation between the XTD2 and XTD9 XGM pulse energy measurements is shown in Fig. 5. The slope of the linear fit is 0.97 indicating a high beamline transmission of $97 \%$. The pulse energy data are deduced from the averaged ion signal of the corresponding XGMDh chambers and each pulse train consists of 60 single-shots.

For the correlation in Fig. 5, the XTD2 and XTD9 XGMs were operated with xenon. In order to test our XGMs and the evaluating software, we kept the XTD2 XGM under xenon and changed the gas in XTD9 to krypton. The server in XTD9 automatically changed the cross section and ion mean charge from xenon to krypton. Thereafter, the relation between intensity measured in XTD2 versus intensity measured in XTD9 stood the same. We measured relative intensities in order to remove the SASE fluctuations. Therefore, the gas change was successfully tested. 


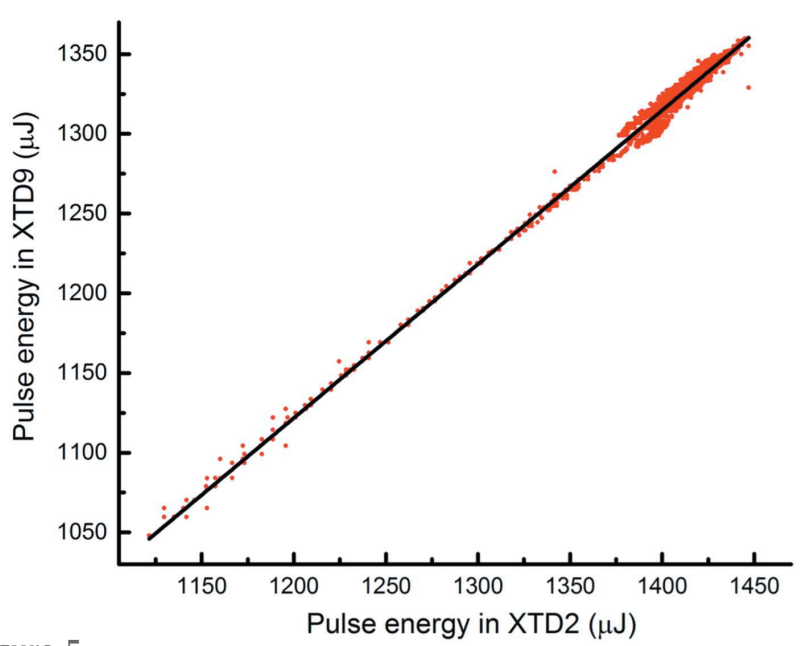

Figure 5

Simultaneous pulse energy measurements for the SASE1 beam at XTD2 and XTD9 XGM. Note that these are averaged energy measurements and not single-shot correlations like in Fig. 8. The slope of the linear fit is 0.97 indicating a beamline transmission of $97 \%$ (no filters inserted). Data sources are XTD2 and XTD9 XGMs (SASE1), averaged ion signals of XGMDh chambers, date 15 June 2018, wavelength $0.134 \mathrm{~nm}, 60$ pulses per train, both XGMs with Xe gas targets. The absolute measurement uncertainty $\sigma$ of the energy was $7-10 \%$ for both XGMs.

While we operated the XTD9 XGM with krypton, the absolute measurement uncertainty $\sigma$ increased in single-pulse mode from $9-12 \%$ to $34-37 \%$. The reason for this is that at $0.134 \mathrm{~nm}$ xenon has a higher cross section than krypton, which leads to higher ion currents with a smaller error. With increasing pulse number per train the uncertainty with krypton becomes closer and closer to the xenon operation. For example, already at two pulses per train the uncertainty $\sigma$ for xenon and krypton is $8-11 \%$ and $28-31 \%$, respectively.

In addition to the pulse energy measurements in XTD2 and XTD9, one can also obtain the simultaneous beam positions in the horizontal and vertical direction. These correlations are shown in Fig. 6 for the horizontal (left) and vertical (right) directions. These data are from the same run as for Fig. 5 and they are also deduced from the averaged ion signal of the corresponding XGMDh and XGMDv chambers. Note that the slopes of the linear fits are considerably greater than 1 . We expect a slope of unity if the SASE beam shows only plane parallel offsets. These larger slopes of 3.8 and 2.5 for the horizontal and vertical direction, respectively, indicate pointing jitter. Since the XTD9 XGM is $878 \mathrm{~m}$ away from the source point, a beam deviation of $0.4 \mathrm{~mm}$, as seen in the horizontal direction, corresponds to $0.5 \mu \mathrm{rad}$. In the future, this non-invasive position measurement at the XTD9 XGM will be used as an input for an active feedback system to the mirrors.

Additionally, we inserted a pop-in imager type I (Koch et $a l ., 2015,2019$ ) into the beam in the SPB branch of XTD9 and compared the XGM position measurement with the centre of mass of the XFEL spot on the screen. With an upstream mirror we moved the beam in the beam direction from the right (positive values) to the left (negative values) and compared the steps in millimetres that are measured with the XGM and imager (see Fig. 7). Note that the imager (pixels to millimetres) and XGM (current to millimetres) are calibrated independently. Only a linear offset to the imager position data was added for a better visualization. While the imager follows the beam steps immediately, the XGM needs tens of seconds averaging time of the ion signal to follow, but thereafter the steps are identical. The right image in Fig. 7 shows the correlation between the position measurement of the imager and the XGM where the linear fit slope is almost unity. This is reasonable since the imager and XGM are spatially very close at this beamline. Note that the imager data show at the beginning of the mirror movement a short deflection in the opposite direction. This could be related to the mirror itself when it starts moving.

We repeated this measurement at the HED branch of XTD6 (SASE2) and found a slope of 0.83. Here the distance between the XGM and imager is much larger than in the previous example: The tunnel positions from the injector are $2785 \mathrm{~m}$ for the mirror, $3261 \mathrm{~m}$ for the XGM and $3334 \mathrm{~m}$ for the
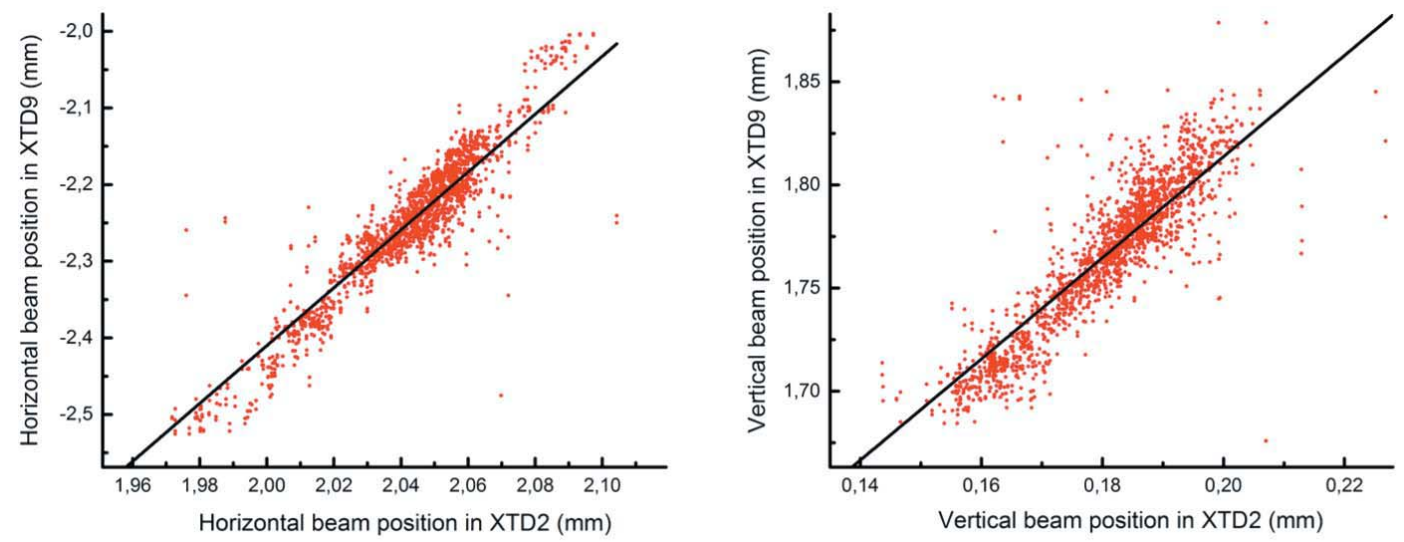

Figure 6

Simultaneous beam position measurements for the SASE1 beam at XTD2 and XTD9 XGM. Horizontal (left graph) and vertical (right graph) beam position correlations are shown. The horizontal and vertical slope of the linear fits are 3.8 and 2.5, respectively. Data sources are XTD2 and XTD9 XGMs (SASE1), averaged ion signals of XGMDh and XGMDv chambers, date 15 June 2018, wavelength $0.134 \mathrm{~nm}$, 60 pulses per train, both XGMs with Xe gas targets. The distance between the SASE1 source point and XTD2 and XTD9 XGM is $209 \mathrm{~m}$ and $878 \mathrm{~m}$, respectively. 

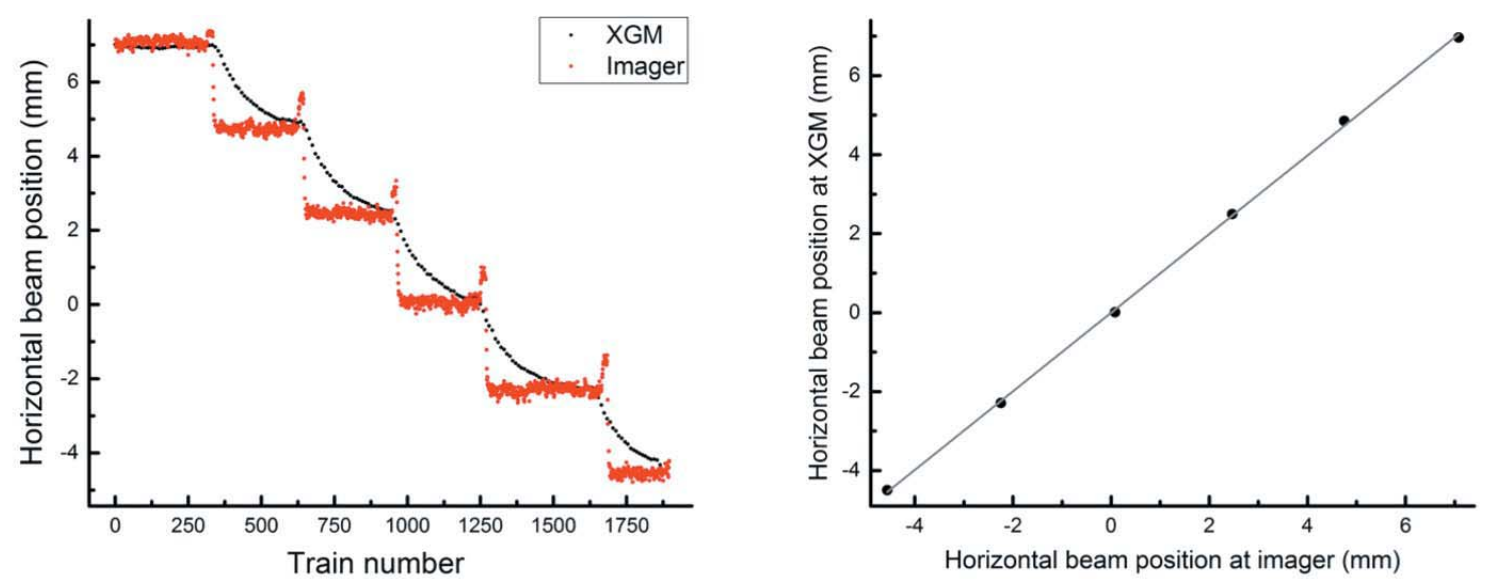

Figure 7

Simultaneous beam position measurements of imager pop-in type I and XGM in the SPB branch of XTD9. (Left) Five discrete steps evaluated out of imager (red dots) and XGM (black dots) position data. Imager position data were linearly shifted with respect to the XGM data for better visualization. (Right) Correlation between imager and XGM position data after XGMD ion signal is settled. The slope of the linear fit is 0.996 with a standard deviation of 0.009. The data source is XTD9 XGM (SASE1), pulse energy at XTD2 XGM was $400 \mu \mathrm{J}$, attenuated to $100 \mu \mathrm{J}$ at XTD9, averaged ion signal of XGMDh, date 24 August 2017, wavelength $0.136 \mathrm{~nm}$, XGM with a Xe gas target.

imager. Since the mirror movement changes the pointing of the beam, we calculate a slope of 0.87 which is close to the measured value.

At the end of this section we want to present single-shot pulse-energy correlation measurements, which are shown in Fig. 8. SASE3 was running at 120 pulses per train at $1.1 \mathrm{keV}$ photon energy and the gas absorber was set to a transmission of $5.5 \%$. All 120 intra-bunch pulses show a linear correlation

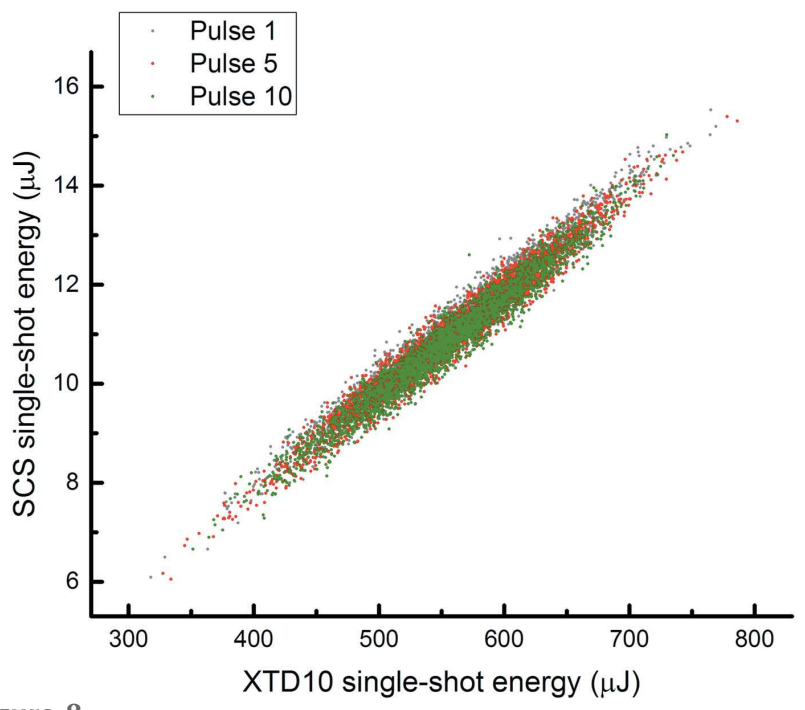

Figure 8

Single-shot pulse energy correlation for the SASE3 beam at the XTD10 and SCS XGM. Correlation of single-shot intra-pulse-train numbers 1, 5 and 10 are shown. The correlation coefficient for the intra-pulse number 1 is 0.98 and the scatter points stay within $\pm 7 \%$ (lower absolute measurement uncertainty). The standard deviation of the SASE fluctuation for this run is measured consistently with both XGMs to be around $11 \%$. The slopes are 0.02 indicating an overall beamline and gas attenuator transmission to the SCS hutch of $2 \%$ for these settings. Data sources are the single-shot XGMDh electron signals calibrated to the average ion signal, date 11 November 2018, wavelength $1.127 \mathrm{~nm}, 120$ pulses per train, XTD10 XGM with Kr and SCS XGM with Xe gas target, gas attenuator was set to $5.5 \%$ transmission. The absolute measurement uncertainty $\sigma$ for both XGMs was $7-10 \%$ between the XTD10 and SCS XGM. For clarity, only singleshot linear correlations of intra-pulse numbers 1, 5 and 10 are shown in Fig. 8. The linear slope is 0.02 indicating an overall gas absorber and beamline transmission to the SCS experiment for this special case of $2 \%$. The correlation coefficient for the intra pulse number 1 is 0.98 and the scatter points stay within $\pm 7 \%$ which is the lower absolute measurement uncertainty of the XGMs. The distance separating the XGMs of $211 \mathrm{~m}$ and the five mirrors in between are further potential sources of scatter. For XTD10 and SCS the mean pulse energy values are $563 \mu \mathrm{J}$ and $11.2 \mu \mathrm{J}$ and the standard deviations are $61 \mu \mathrm{J}$ and $1.26 \mu \mathrm{J}$, respectively. Therefore, for this run the standard deviation of the SASE fluctuation was measured with both XGMs to be around $11 \%$.

\section{Summary and outlook}

We have demonstrated operation of XGMs in the SASE1, SASE2 and SASE3 beamlines of the European XFEL. We presented non-invasive average and single-shot pulse energy measurements and energy correlation results between different XGMs. Simultaneous averaged beam position monitoring in the direct undulator beam and at the end of the tunnel has been realized. Pre-installation calibrations were successfully cross-checked by position correlations between XGMs and imagers.

The non-invasive beam position monitoring will be used in the future as input for mirror feedbacks to stabilize the beam at the experiment. Currently, the XGM operating system software is updated to deal with the so-called fresh bunch mode of operation, where dedicated pulses are addressed for lasing in SASE1 but suppressed for lasing in SASE3 and vice versa. Last but not least, we will explore in the future a wider parameter space for the XGMs, including photon energies beyond the initial ranges of 9-14 keV (SASE1) and 530$1200 \mathrm{eV}$ (SASE3) and repetition rates of up to 2700 pulses. 


\section{Acknowledgements}

An XGM is a very elaborate instrument which needs support from several experts. The authors thank Sören Grunewald for software development, Susanne Bonfigt, Maciej Brachmanski and Bican Yilmaz for the vacuum assembly and hardware implementation, Torben Falk, Johannes Risch and Sonay Sayar for cabling, and Peter Göttlicher and Narcisse Ngada for their advice about grounding. We appreciate the collaboration with the machine operators in the European XFEL control room. We thank Vladimir Rybnikov, Olaf Hensler, Tim Wilksen, Raimund Kammering and Lars Fröhlich for their ongoing DOOCS control development including DAQ implementation. Additionally, we thank several groups for their constructive cooperation, like DESY MEA1 and BAU12 for transport, MEA2 for survey and alignment, and the European XFEL groups AE, CAS, ITDM, Vacuum, SCS, SPB, HED, EET and TS.

\section{References}

Buck, J. (2012). Conceptual Design Report: Online Time-of-Flight Photoemission Spectrometer for X-ray Photon Diagnostics, doi:10.3204/XFEL.EU/TR-2012-002.

Frühling, U., Wieland, M., Gensch, M., Gebert, T., Schütte, B., Krikunova, M., Kalms, R., Budzyn, F., Grimm, O., Rossbach, J. \& Plönjes, E. (2009). Nat. Photon. 3, 523-528.

Grünert, J., Carbonell, M. P., Dietrich, F., Freund, W., Koch, A., Kujala, N., Laksman, J., Liu, J. \& Maltezopoulos, T. (2019a). AIP Conf. Proc 2054, 030014.

Grünert, J. Hauf, S., Heisen, B., Aplin, S., Beg, M., Bergemann, M., Bondar, V., Boukhelef, D., Danilevsky, C., Wajid, E., Essenov, S., Fabbri, R., Flucke, G., Fulla Marsa, D., Göries, D., Giovanetti, G., Hickin, D., Jarosiewicz, T., Kamil, E., Khakhulin, D., Klimovskaia, A., Kluyver, T., Kirienko, Y., Kuhn, M., Maia, L., Mamchyk, D., Mariani, V., Mekinda, L., Michelat, T., Münnich, A., Padee, A., Parenti, A., Santos, H., Silenzi, A., Teichmann, M., Weger, K., Wiggins, J., Wrona, K., Xu, C., Youngman, C., Zhu, J., Fangohr, H. \& Brockhauser, S. (2019b). J. Synchrotron Rad. 26. To be published.

Hauf, S., Heisen, B., Aplin, S., Beg, M., Bergemann, M., Bondar, V., Boukhelef, D., Danilevsky, C., Wajid, E., Essenov, S., Fabbri, R., Flucke, G., Fulla Marsa, D., Göries, D., Giovanetti, G., Hickin, D., Jarosiewicz, T., Kamil, E., Khakhulin, D., Klimovskaia, A., Kluyver, T., Kirienko, Y., Kuhn, M., Maia, L., Mamchyk, D., Mariani, V., Mekinda, L., Michelat, T., Münnich, A., Padee, A., Parenti, A., Santos, H., Silenzi, A., Teichmann, M., Weger, K., Wiggins, J., Wrona, K., Xu, C., Youngman, C., Zhu, J., Fangohr, H. \& Brockhauser, S. (2019). J. Synchrotron Rad. 26. To be published.

Kato, M., Saito, N., Tiedtke, K., Juranić, P. N., Sorokin, A. A., Richter, M., Morishita, Y., Tanaka, T., Jastrow, U., Kroth, U., Schöppe, H., Nagasono, M., Yabashi, M., Tono, K., Togashi, T., Kimura, H., Ohashi, H. \& Ishikawa, T. (2010). Metrologia, 47, 518-521.

Kato, M., Tanaka, T., Kurosawa, T., Saito, N., Richter, M., Sorokin, A. A., Tiedtke, K., Kudo, T., Tono, K., Yabashi, M. \& Ishikawa, T. (2012). Appl. Phys. Lett. 101, 023503.

Koch, A., Freund, W., Grünert, J., Planas, M., Roth, T., Samoylova, L. \& Lyamayev, V. (2015). Proc. SPIE, 9512, 95121R.

Koch, A., Johannes, R., Freund, W., Maltezopoulos, T., Planas, M. \& Grünert, J. (2019). J. Synchrotron Rad. 26. To be published.

Laksman, J., Buck, J., Glaser, L., Planas, M., Dietrich, F., Liu, J., Maltezopoulos, T., Scholz, F., Seltmann, J., Hartmann, G., Ilchen, M., Freund, W., Kujala, N., Viefhaus, J. \& Grünert, J. (2019). J. Synchrotron Rad. 26, 1010-1016.
Lutman, A. A., MacArthur, J. P., Ilchen, M., Lindahl, A. O., Buck, J., Coffee, R. N., Dakovski, G. L., Dammann, L., Ding, Y., Dürr, H. A., Glaser, L., Grünert, J., Hartmann, G., Hartmann, N., Higley, D., Hirsch, K., Levashov, Y. I., Marinelli, A., Maxwell, T., Mitra, A., Moeller, S., Osipov, T., Peters, F., Planas, M., Shevchuk, I., Schlotter, W. F., Scholz, F., Seltmann, J., Viefhaus, J., Walter, P., Wolf, Z. R., Huang, Z. \& Nuhn, H.-D. (2016). Nat. Photon. 10, 468 472.

Maltezopoulos, Th., Cunovic, S., Wieland, M., Beye, M., Azima, A., Redlin, H., Krikunova, M., Kalms, R., Frühling, U., Budzyn, F., Wurth, W., Föhlisch, A. \& Drescher, M. (2008). New J. Phys. 10, 033026.

Moeller, S., Arthur, J., Brachmann, A., Coffee, R., Decker, F., Ding, Y., Dowell, D., Edstrom, S., Emma, P., Feng, Y., Fisher, A., Frisch, J., Galayda, J., Gilevich, S., Hastings, J., Hays, G., Hering, P., Huang, Z., Iverson, R., Krzywinski, J., Lewis, S., Loos, H., Messerschmidt, M., Miahnahri, A., Nuhn, H., Ratner, D., Rzepiela, J., Schultz, D., Smith, T., Stefan, P., Tompkins, H., Turner, J., Welch, J., White, B., Wu, J., Yocky, G., Bionta, R., Ables, E., Abraham, B., Gardener, C., Fong, K., Friedrich, S., Hau-Riege, S., Kishiyama, K., McCarville, T., McMahon, D., McKernan, M., Ott, L., Pivovaroff, M., Robinson, J., Ryutov, D., Shen, S., Soufli, R. \& Pile, G. (2011). Nucl. Instrum. Methods Phys. Res. A, 635, S6-S11.

Richter, M., Gottwald, A., Kroth, U., Sorokin, A. A., Bobashev, S. V., Shmaenok, L. A., Feldhaus, J., Gerth, Ch., Steeg, B., Tiedtke, K. \& Treusch, R. (2003). Appl. Phys. Lett. 83, 2970-2972.

Saito, N., Juranić, P., Kato, M., Richter, M., Sorokin, A., Tiedtke, K., Jastrow, U., Kroth, U., Schoppe, H., Nagasono, M., Yabashi, M., Tono, K., Togashi, T., Kimura, H., Ohashi, H. \& Ishikawa, T. (2010). Metrologia, 47, 21-23.

Saldin, E. L., Schneidmiller, E. A. \& Yurkov, M. V. (2000). The Physics of Free Electron Lasers. Berlin: Springer.

Song, S., Alonso-Mori, R., Chollet, M., Feng, Y., Glownia, J. M., Lemke, H. T., Sikorski, M., Zhu, D., Moeller, S., Lee, H. J., Hunter, M. S., Carini, G., Tiedtke, K., Jastrow, U., Sorokin, A., Richter, M., Owada, S., Tono, K., Saito, N., Tanaka, T., Kato, M., Yabashi, M. \& Robert, A. (2019). J. Synchrotron Rad. 26, 320-327.

Sorokin, A. A. (2004). AIP Conf. Proc. 705, 557-560.

Sorokin, A. A., Jastrow, U., Bonfigt, S. \& Tiedtke, K. (2011). XGMD Final Design, European XFEL and DESY Internal Report. Alfresco Document Id: 19789. European XFEL/DESY, Hamburg, Germany.

Sorokin, A. A., Bican, Y., Bonfigt, S., Brachmanski, M., Braune, M., Jastrow, U. F., Gottwald, A., Kaser, H., Richter, M. \& Tiedtke, K. (2019). J. Synchrotron Rad. 26, 1092-1100.

Tiedtke, K., Feldhaus, J., Hahn, U., Jastrow, U., Nunez, T., Tschentscher, T., Bobashev, S. V., Sorokin, A. A., Hastings, J. B., Möller, S., Cibik, L., Gottwald, A., Hoehl, A., Kroth, U., Krumrey, M., Schöppe, H., Ulm, G. \& Richter, M. (2008). J. Appl. Phys. 103, 094511.

Tiedtke, K., Sorokin, A. A., Jastrow, U., Juranić, P., Kreis, S., Gerken, N., Richter, M., ARP, U., Feng, Y., Nordlund, D., Soufli, R., Fernández-Perea, M., Juha, L., Heimann, P., Nagler, B., Lee, H. J., Mack, S., Cammarata, M., Krupin, O., Messerschmidt, M., Holmes, M., Rowen, M., Schlotter, W., Moeller, S. \& Turner, J. J. (2014). Opt. Express, 22, 21214-21226.

Tschentscher, T., Bressler, C., Grünert, J., Madsen, A., Mancuso, A., Meyer, M., Scherz, A., Sinn, H. \& Zastrau, U. (2017). Appl. Sci. 7, 592.

Villanueva, R. (2014). European XFEL Users Meeting 2014, 29-31 January 2014, Hamburg, Germany, https://xfel.tind.io/ record $/ 1764$ ? $\ln =$ en.

Villanueva, R. (2016). 9th Mechanical Engineering Design of Synchrotron Radiation Equipment and Instrumentation (MEDSI 2016), 11-16 September 2016, Barcelona, Spain, https://xfel.tind.io/ record $/ 1765 ? \ln =$ en. 